\title{
Biosynthesis of Silver Nanoparticles Using Licorice Extract and Evaluation of their Antioxidant Activity
}

\begin{abstract}
ANDREIA CORCIOVA ${ }^{1}$, ANA FLAVIA BURLEC ${ }^{2 *}$, ANA MARIA GHELDIU ${ }^{2}$, ADRIAN FIFERE ${ }^{3 *}$, ANA LACRAMIOARA LUNGOCI ${ }^{3}$, NARCISA MARANGOCI ${ }^{3 *}$, CORNELIA MIRCEA ${ }^{1}$

${ }^{1}$ Grigore T. Popa University of Medicine and Pharmacy, Faculty of Pharmacy, 16 Universitatii Str., lasi, Romania

Iluliu Hatieganu University of Medicine and Pharmacy, Faculty of Pharmacy, 12 Ion Creanga Str., Cluj Napoca, Romania 3Petru Poni Institute of Macromolecular Chemistry, Center of Advanced Research in Bionanoconjugates and Biopolymers, $41 \mathrm{~A}$ Grigore Ghica Voda Alley, 700487, Iasi, Romania

This paper presents a simple and eco-friendlymethod for the synthesis of silver nanoparticles (AgNPs) using licorice (Glycyrrhiza glabra) root extract and the evaluation of their antioxidant activity. UV-Vis spectroscopy, FTIR, TEM and EDX methods were used to characterize the AgNPs. The UV-Vis spectrum shows the characteristic surface plasmon resonance peak at $430 \mathrm{~nm}$. The synthesized AgNPs have a spherical shape and are capped by biomolecules. Furthermore, FTIR spectra and EDX analysis demonstrate the presence of phytochemicals from extract which act as reducing and stabilizing agents. The presence of elemental silver was demonstrated by the peak at $3 \mathrm{keV}$ from EDX spectra. The results following the DPPH free radical scavenging assay suggest that AgNPs present a better antioxidant activity compared to that of the extract. Therefore, the obtained results confirm that licorice represents a source of biomolecules that can be used for synthesizing AgNPs with antioxidant potential.
\end{abstract}

Keywords: licorice, green synthesis, silver nanoparticles, antioxidant activity

Preparation of metal nanoparticles, in particular noble metals nanoparticles, is an emerging field that has gained importance in the last years in various areas including medicine, biology, chemistry and physics. Among these, silver nanoparticles (AgNPs) have been noted for important biomedical applications varying from antimicrobial, antioxidant, hepatoprotective, anti-inflammatory activities to cancer control [1]. Generally, synthesis of nanoparticles can be accomplished by applying physical methods (ultrasonication, irradiation, microwave), chemical methods (chemical reduction, solgel method) or biological methods (using microorganisms, plants). Among the above mentioned methods, the use of plants in the synthesis of AgNPs has recently attracted particular attention due to the environmental aspects of the method [2].

Glycyrrhiza glabra (licorice, sweet wood) is one of the plants with a rich ethnobotanical history, used in traditional medicine since ancient times [3, 4]. Licorice contains important bioactive compounds that belong to classes such as flavonoids, saponins, alkaloids, phenolic compounds, tannins, chalcones, amino acids (asparagine), bitter principles, volatile compounds, mineral salts, polysaccharides, protein, resins, simple sugars, starch, sterols [3-5].

Literature survey has shown that licorice has several important therapeutic properties, such as antibacterial, hepatoprotective, antitussive, antimalarial, antihyperglycemic, antiviral, immunostimulatory, antiinflammatory, expectorant, antiulcer, antitumor, antiallergic, anticoagulant and antidiabetic [3-6].

The present study proposes a new method for the synthesis of AgNPs using licorice root extract. The obtained AgNPs were characterized using UV-Vis spectroscopy, FTIR, EDX and TEM, and their antioxidant activity was evaluated and compared to that of the initial extract.

\section{Experimental part \\ Materials}

Commercially available plant material (licorice roots) was utilized for the preparation of the extract. All reagents used were of analytical grade.

\section{Preparation of licorice root extract}

The licorice root extract was prepared by mixing $10 \mathrm{~g}$ of root fragments with $100 \mathrm{ml}$ water at $40^{\circ} \mathrm{C}$, for 30 minutes.

\section{Phytochemical analysis of licorice root extract}

The total phenolic content. The total phenolic content was determined using the Folin-Ciocalteu method [7]. Briefly, a proper dilution of the extract was mixed with the Folin-Ciocalteu reagent and then a $7.5 \% \mathrm{Na}_{2} \mathrm{CO}_{3}$ solution was added. The absorbance was measured at $760 \mathrm{~nm}$, after a period ranging from 30 seconds to 8 minutes. The total phenolic content was expressed as mg gallic acid equivalents per gram of dried plant material (mg GAE/g plant).

\section{The total flavonoid content}

The total flavonoid content was determined using the aluminum chloride colorimetric method [7] that is based on the reaction with aluminum chloride $25 \mathrm{~g} / /$ in the presence of sodium acetate $100 \mathrm{~g} / \mathrm{l}$. The absorbance was determined after 15 minutes at $430 \mathrm{~nm}$. The total flavonoid content was expressed as mg quercetin equivalents per gram of dried plant material ( $\mathrm{mg} \mathrm{QE} / g$ plant).

\section{The total phenolic acids content}

The concentration of total phenolic acids was estimated using a method officialized by the European Pharmacopoeia [8] in which the extract is treated with 0.5 $\mathrm{M} \mathrm{HCl}$, a solution that contains sodium nitrite and sodium molybdate and with $\mathrm{NaOH} 8.5 \%$. The absorbance was

\footnotetext{
*email: flavia_burlec@hotmail.com; fifere@icmpp.ro; nmarangoci@icmpp.ro
} 
measured at $525 \mathrm{~nm}$. The percentage of phenolic acids was expressed as mg chlorogenic acid equivalents per gram of dried plant material (mg CAE/g plant).

Quantification of polyphenols: For the quantification of polyphenols a HPLC-UV-MS method was used [9]. Briefly, compounds were separated on a Zorbax SB-C18 reversedphase analytical column $100 \mathrm{~mm} \times 3.0 \mathrm{~mm}$ i.d., $3.5 \mu \mathrm{m}$ particles, using as mobile phase methanol : acetic acid $0.1 \%(\mathrm{v} / \mathrm{v})$, in gradient elution. For separation an Agilent 1100 HPLC series system (Agilent, USA) was used, while for identification an Agilent Ion Trap 1100 VL mass spectrometer with Turbo-lon spray (ESI) interface in negative ion mode was used. Quantification was performed using UV detection at $330 \mathrm{~nm}$ and $370 \mathrm{~nm}$. Apigenin, caffeic acid, chlorogenic acid, p-coumaric acid, fisetin, hyperoside, isoquercitrin, kaempferol, quercetin, quercitrin, myricetin, rutin (Sigma, Germany), ferulic, gentisic, sinapic acids, luteolin, patuletin (Roth, Germany) and caftaric acid (Dalton, USA) were used as standards.

\section{Synthesis of silver nanoparticles (AgNPs)}

The synthesis of AgNPs was achieved by mixing licorice root extract $(1 \mathrm{ml})$ with a $5 \mathrm{mM} \mathrm{AgNO}$ solution $(9 \mathrm{ml})$, followed by stirring at $600 \mathrm{rpm}$, at $60^{3} \mathrm{C}$. AgNPs were separated by repeated centrifugation at 10,000 rpm for 20 minutes, after which the pellets were dispersed in distilled water and centrifuged to remove the water-soluble compounds, the operation being repeated 3 times. The finally obtained pellets were dried and used for characterization.

\section{Characterization of AgNPs}

UV-Vis spectra of samples were recorded using a J asco $\checkmark 530$ double beam UV-Vis spectrophotometer, in the 300$600 \mathrm{~nm}$ wavelength range and at $1000 \mathrm{~nm} \mathrm{~min}{ }^{-1}$ scanning speed.

Fourier transform infrared (FTIR) spectra were obtained using a Bruker Vertex 70 instrument. The samples were prepared using potassium bromide $(\mathrm{KBr})$ discs. The spectra were registered in the $4000-310 \mathrm{~cm}^{-1}$ range.

Transmission electron microscopy (TEM) analysis was performed with a Hitachi High-Tech HT 7700 Transmission Electron Microscope that operates at $100 \mathrm{kV}$. The samples were placed on a carbon-coated copper grid.

Energy dispersive X-ray analysis (EDX) was carried out by using a Quanta 200 Environmental Scanning Electron Microscope (ESEM). The samples were placed on aluminum supports. For this analysis, an EDX silicon-drift detector was used.

Photon correlation spectroscopy (PCS) and Dynamic light scattering (DLS) were performed using Delsa Nano Submicron Particle Size Analyzer (Beckman Colter).

\section{The antioxidant activity of AgNPs}

The antioxidant activity of AgNPs and of the extract was determined by DPPH free radical scavenging assay [10, 11]. Briefly, the absorbance of the DPPH (2,2-diphenyl-1picrylhydrazyl) solution was registered at $517 \mathrm{~nm}$ before and after adding the nanoparticles or extract and then the percentage of DPPH radical scavenging activity was calculated.

\section{Results and discussions}

\section{Phytochemical analysis}

Secondary plant metabolites such as phenolic acids, flavones, alkaloids and terpenoids are potential precursors in the synthesis of nanoparticles [12]. Therefore, in order to investigate the potential of our extract to synthesize
AgNPs, we determined the total content of phenolic compounds, flavonoids and phenolic acids using spectrophotometric methods.

The following results were obtained after the analyses: total phenolic content $-25.78 \mathrm{mg} \mathrm{GAE} / \mathrm{g}$ plant, total flavonoids content $-3.12 \mathrm{mg}$ QE/g plant and total phenolic acids content $-1.62 \mathrm{mgCAE} / \mathrm{g}$ plant. The values reported in literature for similar extracts vary as follows: total phenolic content - $5.83 \mathrm{mg} \mathrm{GAE} / 100 \mathrm{mg}$ lyophilized infusion, total flavones and flavonols - $0.70 \mathrm{mg}$ QE/100 mg lyophilized infusion [13] or the total phenolic compounds $-4.8 \mathrm{mg} /$ $100 \mathrm{mg}$ dry extract, the flavonoids content expressed as catechins - $2.3 \mathrm{mg} / 100 \mathrm{mg}$ dry extract [14]. Differences can occur due to various sources of plant material (licorice roots), the influence of the harvesting period, the extraction conditions etc.

Moreover, using a HPLC-UV-MS method, the presence of caffeic, p-coumaric and ferulic acids, quercetin and apigenin in the extract was demonstrated. The following compounds were also quantified: caffeic acid $2.61 \mu \mathrm{g} / \mathrm{g}$ plant, p-coumaric acid $7.32 \mu \mathrm{g} / \mathrm{g}$ plant and ferulic acid 2.53 $\mu \mathrm{g} / \mathrm{g}$ plant.

Taking into account the identity and amount of the determined compounds in the extract and their reducing properties, we proceeded to the AgNPs formation process.

\section{Synthesis and Characterization of AgNPs}

Regarding the monitoring of the silver ion reduction reaction, the change in color after mixing the licorice extract with the $\mathrm{AgNO}_{3}$ solution was observed (Figure 1) and the UV-Vis spectra were recorded (Figure 2).

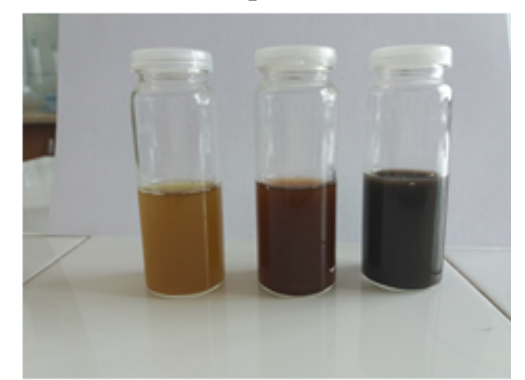

Fig. 1 Pictures of the AgNPs synthesis process in time: initially, after 180 and $600 \mathrm{~min}$

(from left to right)

The reaction proceeding was slow, with no change in color being observed in the first 120 minutes. Afterwards, the yellow color of the initial mixture began to change to brown, which meant that nucleation was initiated and AgNPs formation was observed through the appearance of the specific surface plasmon resonance band at 430 $\mathrm{nm}$ on the UV-Vis spectrum (Fig. 2).

The intensity of the color increased during the reaction time, finally turning into dark brown. Zaheer suggested that the appearance of surface plasmon resonance in the 410-

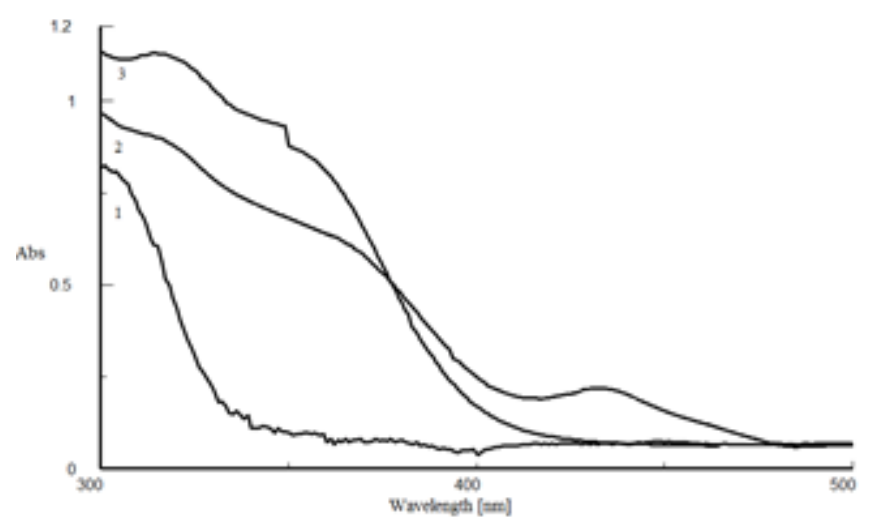

Fig. 2 UV-Vis spectra of $\mathrm{AgNO}_{3}$ solution (1), reaction mixture (2) and starting extract (3) 
$450 \mathrm{~nm}$ range demonstrates that the obtained nanoparticles are spherical [15].

In order to highlight the functional groups of the biomolecules that contribute to AgNPs formation, FTIR spectra of the extract and of the AgNPs were recorded (Figure 3).

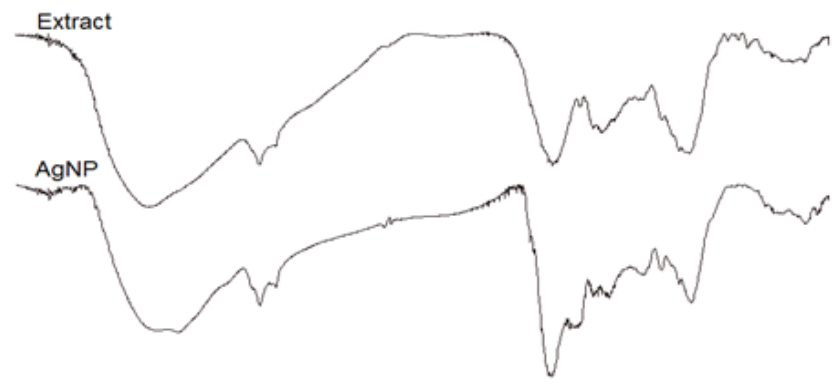

$35003000 \quad 2500 \quad 2000 \quad 1500 \quad 1000$

Fig. 3 Comparative FTIR spectra of licorice extract and AgNPs

Since the spectrum was recorded on a mixture of natural extraction compounds, including polyphenolic compounds and proteins, the FTIR spectrum contains broad absorption bands in which different modes of vibration are superimposed. However, even under these conditions, some fundamental features can be observed, revealing the chemical nature of nanoparticles.

The comparative FTIR spectra show that most peaks found in the extract spectrum are also found in the AgNPs spectrum, which demonstrates the dual role of the compounds present in the extract: reducing and stabilizing agents. Peaks are generally displaced in the AgNPs spectrum. For example: 3419 to $3370 \mathrm{~cm}^{-1}$ characteristic to $\mathrm{OH}$ stretching vibration of hydroxyl group, [16-18], 1629 to $1650 \mathrm{~cm}^{-1}$ attributed to $-\mathrm{C}=\mathrm{C}$ - stretch alkenes [16] which may be overlapped with $\mathrm{C}-\mathrm{N}$ and $\mathrm{C}-\mathrm{C}$ stretching vibrations [17] and $\mathrm{C}=0$ stretching vibration, 1515 to $1512 \mathrm{~cm}^{-1}$ assigned to $C=C$ and $C=0$ groups [18], 1424 to 1392 $\mathrm{cm}^{-1}$ possibly due to $\mathrm{N}-\mathrm{H}$ and C-N stretching vibrations [17] or $-C-C$ stretch in aromatics [16] and 1029 to $1024 \mathrm{~cm}^{-1}$ assigned to $\mathrm{C}-\mathrm{O}$ stretch from alcohols, carboxylic acids, esters [16] or the aliphatic amines [18]. Moreover, the disappearance or decrease in peak intensity in the AgNPs spectrum ranging from $880-700 \mathrm{~cm}^{-1} \mathrm{can}$ be observed. The analysis confirms the presence of some functional groups which may belong to phenolic compounds, flavonoids, proteins; these substances can participate in the formation, capping and stabilization of AgNPs.

The size and morphology of the prepared AgNPs were evaluated by TEM analysis. Figure 4 shows TEM image of the well dispersed AgNPs, confirming the spherical morphology and relative uniformity in size.

The average particle size was found to be around 10-15 $\mathrm{nm}$. Most AgNPs are dispersed, being separated by a layer of organic compounds, as demonstrated by FTIR analysis. The presence of this organic layer confirms the stabilization of the surface of AgNPs [19].

The chemical composition of AgNPs was evaluated by EDX analysis, the spectrum being presented in Figure 5.

The EDX spectrum revealed a peak at $3 \mathrm{KeV}$ absorption, characteristic to metallic silver, due to surface plasmon resonance. The quantitative results of EDX spectra for AgNPs showed a yield of 25.91 (weight \%) silver. Furthermore, the presence of peaks characteristic for elements such as carbon (45.4 weight \%), oxygen (21.32 weigh \%), nitrogen (6.07 weigh \%) demonstrates the presence of biomolecules at the surface of AgNPs [17].

The particle size distribution obtained by DLS shows a polydisperse nature and an average size of $698 \mathrm{~nm}$, suggesting a certain level of aggregation during the stabilization of the dispersed system. In addition, the zeta potential (Figure 6) value is - $29.41 \mathrm{mV}$.

The negative value of the zeta potential demonstrates the stability of AgNPs, shows a good colloidal nature [17] and the capping action of biomolecules [20], suggesting that the heterogeneous system of the aqueous AgNPs dispersion can be stabilized by electrostatic repulsion between nanoparticles due to the surrounding organic layer.

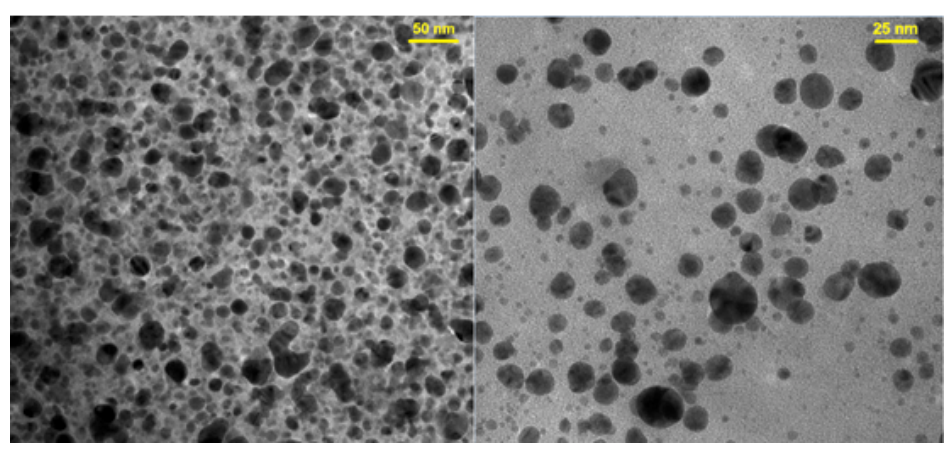

Fig. 4 TEM images of AgNPs

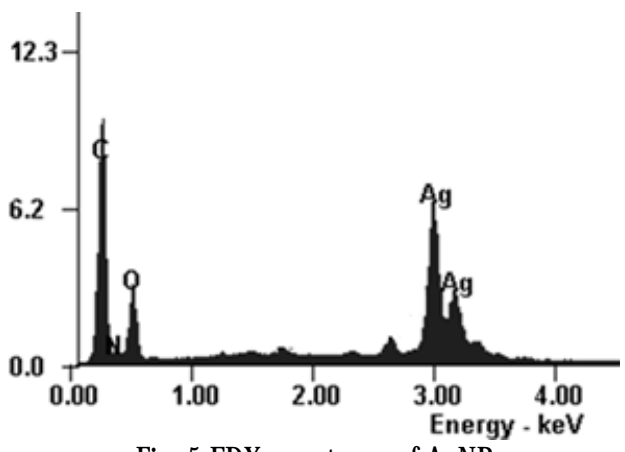

Fig. 5 EDX spectrum of AgNPs

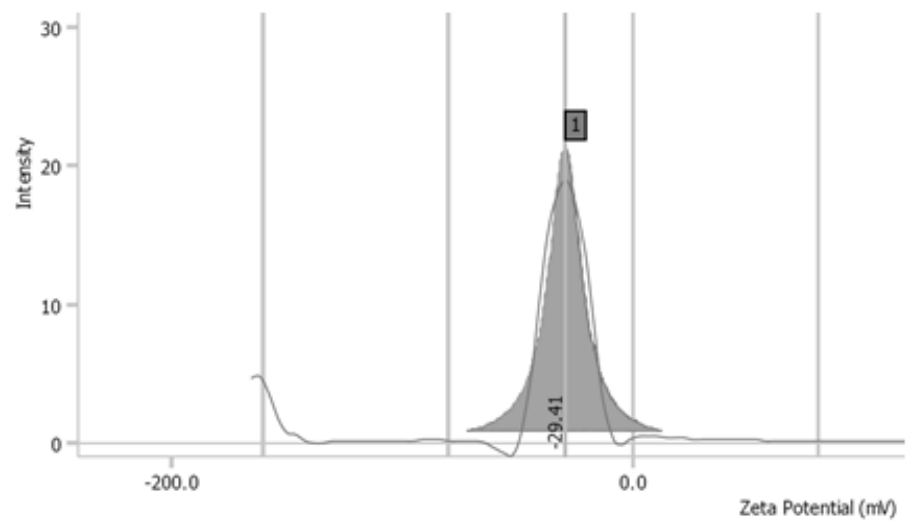

Fig. 6 Zeta potential of AgNPs 


\section{Antioxidant activity of AgNPs}

The antioxidant activity of AgNPs was evaluated using DPPH scavenging method and was compared with that of the licorice extract. A correlation between the concentration of the AgNPs sample and the antioxidant activity was observed. For the extract, the DPPH scavenging activity of the maximum concentration taken into work presents an inhibition smaller than $50 \%$, therefore the $\mathrm{IC}_{50}$ could not be calculated. The $\mathrm{IC}_{50}$ value of AgNPs was $0.37 \pm 0.005 \mathrm{mg} / \mathrm{ml}$. The obtained AgNPs showed effective antioxidant activity against the DPPH radical. These results can be explained by the presence of functional groups belonging to the biomolecules which adhered to them [21].

\section{Conclusions}

In the present study, we described a rapid and environmentally friendly method for the synthesis of AgNPs using a licorice extract. Initially, the extract was evaluated for total content of phenolic compounds, flavonoids and phenolic acids; caffeic, p-coumaric and ferulic acids were quantified. Synthesized AgNPs were characterized by UVVis spectroscopy, FTIR, TEM and EDX techniques, revealing the presence of dispersed spherical nanoparticles containing an organic shell. These nanoparticles exhibita higher scavenging activity when compared to the initial extract. In conclusion, licorice extract represents a good source of biocompounds with reducing capacity that can be successfully used for the synthesis of stabilized spherical silver nanoparticles with potential biological activities.

\section{References}

1. HEYDARI, R., Herb. Med. J., 2, nr. 2, 2017, p. 87

2. CHUNG, I.M., PARK, I., SEUNG-HYUN, K., THIRUVENGADAM, M., RAJAKUMAR, G., Nanoscale. Res. Lett., 11, nr. 40, 2016, p. 1 3. KAUR, R., KAUR, H., DHINDSA, A.S., Int. J. Pharm. Sci. Res., 4, nr. 7, 2013, p. 2470
4. SHARMA, V., AGRAWAL, R.C., Mintage J. Pharm. Med. Sci., 2, nr. 3, 2013, p. 15

5. NESAR, A., NOORUL, H., KHALID, M., JUBER, A., MUJ AHID, M., BADRUDDIN, ANURADHA, M., NAZMA, K. Adv. J Pharm Life Sci Res.,

4, nr. 2, 2016, p. 23

6. DAMLE, M., Int. J. Herb. Med., 2, nr. 2, 2014, p. 132

7. MURESAN, M., BENEDEC, D., VLASE, L., OPREAN, R., TOIU, A., ONIGA, I., Studia UBB Chemia, 60, nr. 1, 2015, p 127

8.*** European Directorate for Quality Medicines, European Pharmacopoeia, $7^{\text {th }}$ Edition, European Directorate for Quality Medicines, Strasbourg, 2010.

9. KHALAF, I., VLASE, L., LAZAR, D, CORCIOVA, A., IVANESCU, M., LAZAR, M.I., Farmacia, 58, nr. 4, 2010, p. 416

10. TATARINGA, G., STAN, C., MIRCEA, C., JITAREANU, A., ZBANCIOC, A.M., Farmacia, 64, nr. 4, 2016, p. 533

11. DRAGAN, M., STAN, C.D., PANZARIU, A., PROFIRE, L., Farmacia, 64, nr. 2, 2016; p. 194

12. KUPPUSAMY, P., YUSOFF, M.M., MANIAM, G.P., GOVINDAN, N., Saudi Pharm. J., 24, nr. 4, 2016, p. 473

13. CHEEL, J., ONOFRE, G., VOKURKOVA, D., TUMOVA, L., NEUGEBAUEROVA, J., Pharmacogn Mag., 6, nr. 21, 2010, p. 26

14. MOHAMED, S.K., Pak. J. Nutr., 13, nr. 5, 2014, p. 267

15. ZAHEER, Z., RAFIUDDIN., Colloids Surf. B., 90, 2012, p. 48

16. THAKUR, D., ABHILASHA, JAIN, A., GHOSHAL, G. J., Sci. Ind. Res., 75, 2016, p. 487

17. JYOTI, K., BAUNTHIYAL, M., SINGH, A., J. Radiat. Res. Appl. Sci., 9, nr. 3, 2016; p. 217

18. BUNGHEZ, I.R., DUMITRESCU, O, SOMOGHI, R., IONITA, I., ION, R.M., Rev. Chim.(Bucharest), 66, no. 8, 2015, p. 1112

19. SHAIK, M.R., KHAN, M., KUNIYIL, M., AL-WARTHAN, A., ALKHATHLAN, H.Z., SIDDIQUI, M.R.J., SHAIK, J.P., AHAMED, A., MAHMOOD, A., KHAN, M., ADIL, S.F. Sustainability, 10, 2018, p. 913

20. RAJ A, S., RAMESH, V., THIVAHARAN, V., Arabian J. Chem., 10, nr. 2, 2017, p. 253

21. BHAKYA, S., MUTHUKRISHNAN, S., SUKUMARAN, M., MUTHUKUMAR, M., Appl. Nanosci., 6, 2016, p. 755

Manuscript received: 17.08 .2018 